\title{
Análisis de las reducciones de productividad debidas a enfermedad en Bogotá D.C, Colombia
}

\author{
Analysis of productivity reduction due to illness in Bogotá D.C., Colombia \\ Carlos Andrés Cuéllar Moreno ${ }^{a}$
}

\begin{abstract}
:
This work is an exploratory analysis of a larger project on productivity and health for Colombia. In first instance, an exhau stive review of the international literature of the subject is made for both "Absenteeism" and "Presenteeism", subsequently the estimation of lost productivity by "Absenteeism" in Bogotá D.C. during the years 2009 - 2018 representing 7\% of the city's GDP, a statistically significant figure. Finally, conclusions, recommendations and a way to deepen more precise estimates on the subject are provided.
\end{abstract}

Keywords:

Productivity, Health, Urban Economics

Resumen:

El presente trabajo es un análisis exploratorio de un proyecto más grande sobre productividad y salud para Colombia. En primera instancia se hace una exhaustiva revisión de la literatura internacional de la temática tanto para "Absenteeism" y "Presenteeism", posteriormente se realiza la estimación de pérdida de productividad por “Absenteeism” en Bogotá D.C. durante los años 2009 - 2018 representando el 7\% del PIB de la ciudad, cifra significativa estadísticamente. Finalmente se brindan conclusiones, recomendaciones y un camino para profundizar en estimaciones cada vez más precisas sobre el particular.

\section{Palabras Clave:}

Productividad, salud y economía urbana

\section{Introducción}

A nivel mundial las pérdidas de productividad debidas a enfermedad laboral han sido estudiadas por Mitchell \& Bates (2011), y en el caso de Estados Unidos se obtuvo (con una muestra de más de 1 millón de participantes) una pérdida de productividad que asciende a 260 billones de dólares anuales para el país. Además, aseguran que este resultado puede llegar a costarle a algunas empresas una mayor proporción que los gastos médicos directos que realizan para sus empleados. Inclusive, si se desagrega por tipo de enfermedad (ver gráfico 1) se evidencia que esta situación no es de poca relevancia a nivel firma y a nivel agregado y que efectivamente resulta muy costoso para las firmas en general.
Gráfico 1. Pérdida en productividad debida a malas condiciones de salud en Estados Unidos

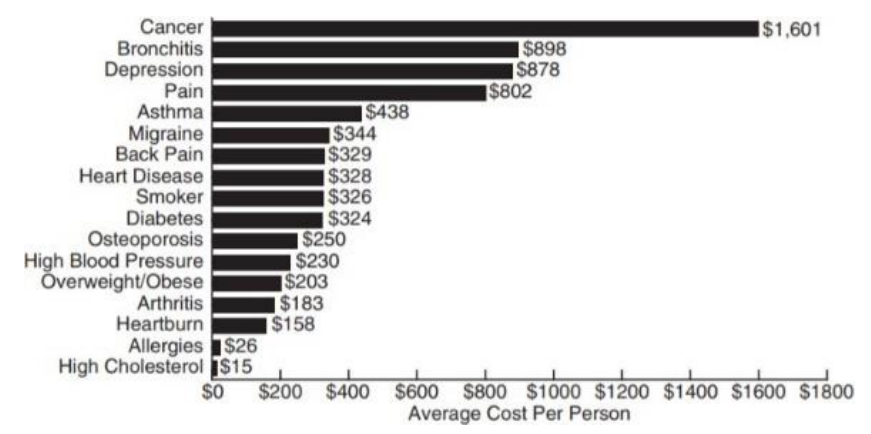

Fuente: Tomado de Mitchell y Bates (2011, p. 97) 
Adicional a lo anterior, Mitchell \& Bates (2011) han obtenido que los participantes reportaron un promedio de 1,99 días de trabajo perdidos debido a enfermedad (Absenteeism), y 9,04 días trabajados con limitación en su rendimiento laboral por la misma condición (Presenteeism). Lo cual les permite inferir que existe una relación inversa entre productividad y malas condiciones generales de salud.

En el caso colombiano, según el Consejo Privado de Competitividad, CPC (2014, p. 90), el país, “... presenta grandes diferencias en la productividad laboral entre sectores (intersectorial), al igual que entre firmas al interior de un mismo sector (intra - sectorial), lo anterior es un claro ejemplo de que los factores productivos no se están asignando a las actividades más productivas, lo que explica los bajos niveles de productividad del país". Al calcular la tasa de crecimiento promedio de la Productividad Total de los Factores (PTF, de aquí en adelante) para el período de interés, el resultado ha sido del $0,3 \%$ (ver gráfico 2) mientras que a finales del siglo $X X$ decreció al 0,29\%; es decir, Colombia presenta un rezago histórico en materia de productividad comparado con economías más desarrolladas; inclusive el gráfico 2, muestra que para el período 2014 - 2017 la situación ha empeorado considerablemente, decreciendo al 0,53\%, y es posible que esta tendencia prevalezca a futuro. Si se toma como referencia a Estados Unidos para el mismo período y la misma periodicidad anual, el resultado ha sido un crecimiento de la PTF de 0,51\%, 0,73\% y $0,53 \%$, respectivamente, lo cual es diametralmente opuesto a Colombia.

Gráfico 2. Productividad total de los factores en Colombia.2009 - 2017

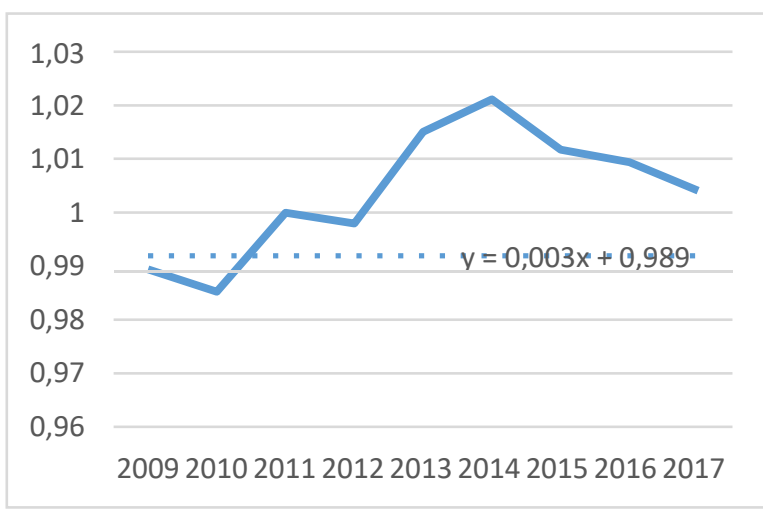

Fuente: Cálculos del autor con base en Penn World Tables
Con esto en mente, si se compara el comportamiento de la PTF a nivel nacional con el de la tasa de crecimiento de enfermedades laborales para el período 2009 - 2018, a saber $1,76 \%$ y $2,02 \%$ en promedio anual para Colombia y Bogotá D.C., y tomando en cuenta que a nivel internacional se ha encontrado una relación inversa entre ambas variables, ver gráficos 2, 3 y 4 . Así también se puede inferir la existencia de una relación entre baja productividad y malas condiciones de salud debido al trabajo. Con esto en mente, si se compara el comportamiento de la PTF a nivel nacional con el de la tasa de crecimiento de enfermedades laborales para el período 2009 - 2018, a saber $1,76 \%$ y $2,02 \%$ en promedio anual para Colombia y Bogotá D.C., y tomando en cuenta que a nivel internacional se ha encontrado una relación inversa entre ambas variables, ver gráficos 2 , se puede inferir preliminarmente la existencia de una relación entre baja productividad y malas condiciones de salud debido al trabajo cuando la causa es el "Absenteeism".

Gráfico 3. Tasa de crecimiento de enfermedades de origen laboral en Colombia. 2009 - 2017

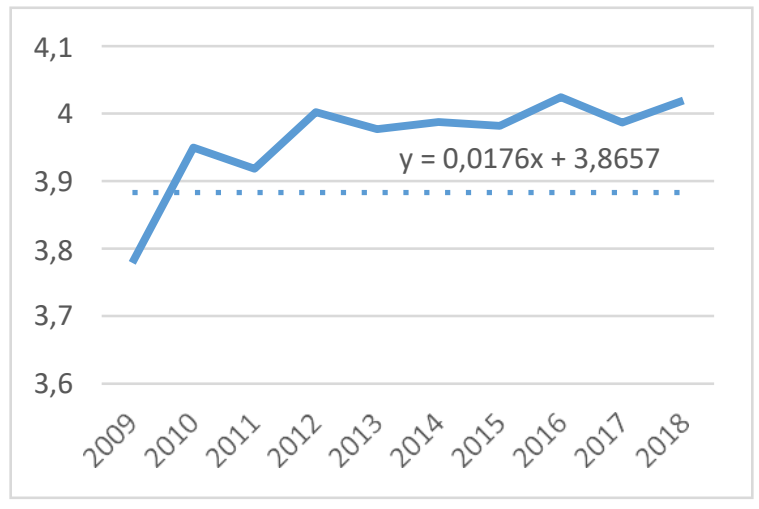

Fuente: Cálculos del autor con base en Penn World Tables9.1. Fuente: Cálculos del autor con base en datos FASECOLDA

\section{Marco Teórico}

\section{'Presenteeism', 'Absenteeism' y Productividad}

Se reconoce internacionalmente que al menos dos conceptos inciden negativamente en la productividad, entendida como la reducción en la "cantidad" y "calidad" de la capacidad de producción por unidad de trabajo por enfermedades laborales mientras se está en la empresa. Lo anterior se denomina "absenteeism"* y "presenteeism" $\dagger$ debido a distintos tipos de enfermedades. Recientemente Zhang, Wei. et al. (2015, p. 62) y Zhang, Wei et al. (2017, p. 3) han demostrado que

\footnotetext{
${ }^{1}$ Datos calculados a partir de la base de datos de Penn world tables. Ver link: https://www.rug.nl/ggdc/productivity/pwt/

Absenteeism: se refiere al acto de no ir a trabajar por una enfermedad laboral certificada. Tomado de:

https://dictionary.cambridge.org/es/diccionario/ingles/absen teeism, consultado el 27 de abril de 2019

'Presenteeism: se refiere al acto de estar más tiempo del usual en el puesto de trabajo para intentar demostrar que se está trabajando duro y que eres importante en el equipo de trabajo, así no se trabaje efectivamente. https://dictionary.cambridge.org/es/diccionario/ingles/absen teeism, consultado el 27 de abril de 2019
} 
es más costoso lo segundo que lo primero tanto para firmas grandes como pequeñas.

Gráfico 4. Tasa de crecimiento enfermedad de origen laboral en Bogotá, 2009 - 2018

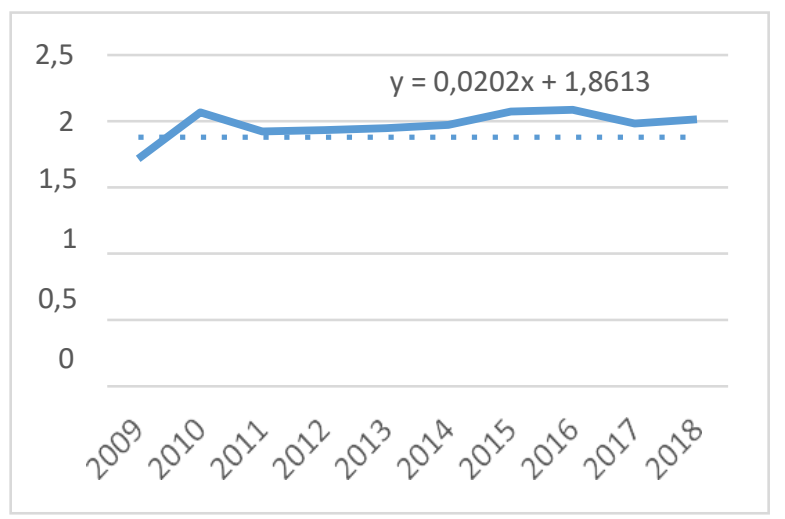

Fuente: Cálculos del autor con base en datos FASECOLDA

Presenteeism': evidencia microeconómica internacional

Al ser el 'presenteeism' un costo para las firmas, es relevante medir el impacto de las enfermedades laborales en el nivel de actividad económica y las eventuales pérdidas de eficiencia económica a este nivel, ver Jakubczyk, M. \& Koń, B. (2017, 377 - 394); siendo oportuno agregar que deben diferenciarse los trabajadores con "Presenteeism" de los "Free-riders" o polizones, al tenerse en cuenta la certificación de la condición clínica del individuo, y así se evitará tener sesgo de selección.

Dado lo anterior y siguiendo a Pearce, A; Et al. (2015, p. 360 ), en esta primera aproximación se contribuirá con elementos de discusión a los hacedores de política regionales y nacionales desde una perspectiva del costo de las enfermedades para la evaluación económica de nuevos medicamentos, aplicaciones y servicios del sistema de seguridad social en Bogotá.

En el caso de las firmas pequeñas existe evidencia microeconómica internacional adicional, ver Zhang, Wei. et al (2015b, p. 70), donde si hay trabajadores con reducciones ocasionales en sus cargas laborales se elevará su productividad frente a trabajadores que no han recibido esta consideración, pero lo contrario se ha validado para firmas grandes. Concluyendo, se puede afirmar que las pérdidas en productividad pueden exceder la tasa de salario si se involucra trabajo en equipo, siendo una forma de encubrir a aquellos trabajadores con problemas de salud pero que no los revelan.
'Absenteeism': evidencia microeconómica internacional

Petrie et al. (2015) estudia las reducciones de productividad con un enfoque microeconómico debidas a la influenza que redunda en 'absenteeism', lo cual en principio parece menos severo que otras enfermedades, pero aquí se concluye que si incide mucho más en la pérdida de horas laborales $(45 \%$ más frente a otras enfermedades) y de pérdida de productividad (6.0 frente a 5.4 horas por mes, significativo al 1\%) frente a enfermedades respiratorias distintas.

En este orden de ideas, Pearce et. al. (2015, p. 363) utiliza otra aproximación metodológica basada en 'capital humano' (HCA) y 'costos de fricción' (FCA), planteando que las pérdidas de productividad debidas a cáncer de cerebro y de cuello son mayores que las asociadas a otros tipos de cáncer pero que hasta el momento la literatura ha medido esas pérdidas de productividad a través del 'absenteeism' y la muerte prematura con un enfoque de 'capital humano'. Aunque aquí se concentra en el enfoque 'Friction cost approach', donde los costos de productividad son entendidos como costos asociados a la pérdida de productividad y los costos de reemplazo debidos a las enfermedades, incapacidad y muerte de personas productivas, Kigozi, J; Et al (2016, p. 31), y se concluye que las pérdidas totales de productividad de empleados para ambas metodologías son de 253.000 Euros con HCA y de 6.800 Euros usando FCA, siendo los mayores factores de incidencia para cada metodología, mortalidad prematura $(38 \%$ del total) y tiempo fuera del lugar de trabajo $(73 \%$ del total), respectivamente.

\section{'Absenteeism' y 'Presenteeism' en conjunto a nivel microeconómico y macroeconómico}

En el caso de las enfermedades de tipo mental suele generarse 'absenteeism' y 'presenteeism' en el lugar de trabajo, llegando a ser considerado un problema de salud pública mundial. Usando una metodología prospectiva, Suzuki et al. (2015, p. 17) analiza a 1.831 trabajadores japoneses de todos los sectores productivos de Japón obteniendo como resultado que una vez se ajusta por edad y género, los trabajadores con altas tasas de 'presenteeism' eran más propensos a terminar ausentándose de su trabajo debido a enfermedades mentales, tales como depresión. A pesar de no ser una muestra balanceada en términos del número de hombres y mujeres, los resultados para las últimas no fueron significativamente distintos que para los primeros. En conclusión, mayor evidencia a favor de la existencia de 'presenteeism' conlleva a tasas más altas de depresión y de 'absenteeism' debida a enfermedades mentales. 
En el caso de las mujeres en posparto, Wickramasinghe, ND et al (2017) realizan un ejercicio para Sri Lanka donde calculando la pérdida de productividad como la suma de los días pérdidos debidos a la incapacidad parcial o total del individuo, teniendo como resultado que la media de pérdida de productividad fue 15 días (S.D. 7,8 días) y la pérdida de productividad por episodio después de ajustar por 'coping strategies' se tiene como resultado que la pérdida de productividad fue de 7,9 días (S.D. 4,4 días).

Tabla 1. Impacto sobre la disminución de expectativa de vida debido a la incapacidad (DALY) generada por tipo de enfermedad

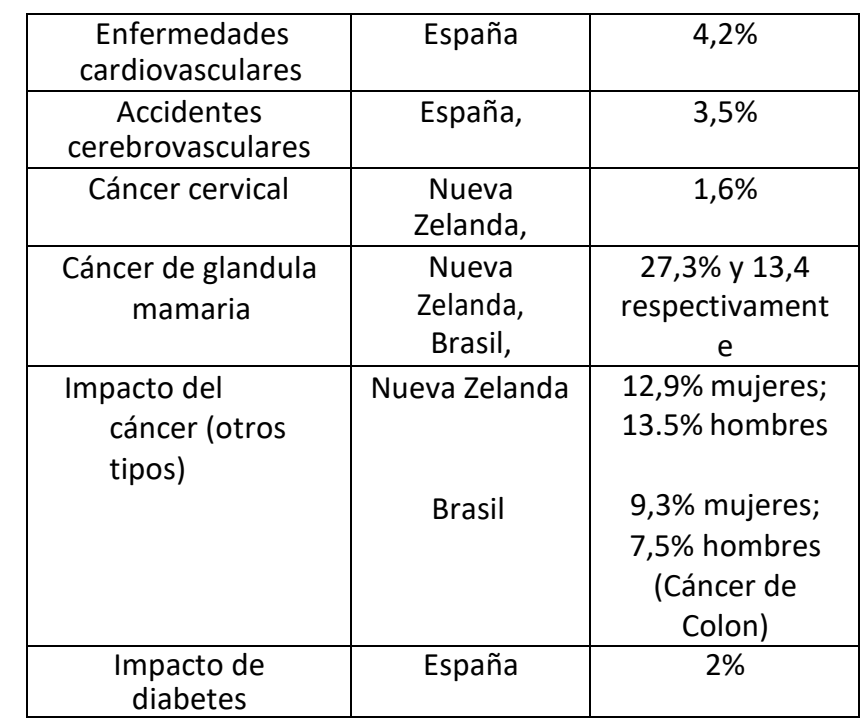

Fuente: Layal et al (2015)

Finalmente a nivel macroeconómico, Layal et al (2015, p. 358 y 366 ) han identificado que las 'non-communicable diseases', ver tabla 1 , presentan profundos impactos económicos a distintos niveles, y concluyen que la prevención y el control de este tipo de enfermedades tiene una incidencia positiva (negativa) en la tasa de crecimiento vía incremento (o pérdida) de productividad; al contribuir al desarrollo científico en el área de la salud y el sistema social como un todo, incrementarse la expectativa de vida y su calidad, pero resaltan que lo más probable es que con los cambios socio - demográficos actuales se incremente la incidencia de este tipo de enfermedades.

\section{Estrategia Empírica}

Para estimar las pérdidas de productividad asociadas a enfermedades laborales, la información disponible en Colombia está en las bases de datos de SISPRO del Ministerio de Salud y Protección Social de Colombia, de riesgos laborales de FASECOLDA y la Encuesta anual manufacturera y de desarrollo e innovación tecnológica del DANE.

En el presente documento siguiendo a Zhang, Wei. et al. (2015) y Zhang, Wei et al. (2017) se entenderá la tasa mensual y trimestral de 'absenteeism' como los días de trabajo pérdidos a causa de una enfermedad laboral certificada y 'presenteeism' como los días de trabajo en que el desempeño laboral del individuo fue bajo por razones de salud debidamente certificados.

Para medir el costo de oportunidad del 'absenteeism' y 'presenteeism', según Mitchell \& Bates (2011) se suele utilizar como variable proxy el nivel de salario promedio por hora de la rama de actividad económica correspondiente y se compara con el número de días laborales perdidos a nivel de empresa. Dado lo anterior, se compara el resultado con los salarios ganados por los empleados que trabajaron a tiempo completo y se halla un multiplicador que se define como el costo que asume un empleador por el 'absenteeism' o 'presenteeism' como una proporción del salario diario que se le hubiese pagado al trabajador enfermo laboralmente por día.

Finalmente, siguiendo a Mitchell y Bates (2011, p. 95) se agregan los resultados y se puede obtener a nivel de rama de actividad económica, municipio, departamento y país, la pérdida mensual, trimestral y anual de productividad debida a enfermedades laborales para el período de interés.

\section{Resultados}

La ciudad de Bogotá ha concentrado aproximadamente el $40 \%$ del total de las empresas del país entre $2009-2018$ y en este lapso de tiempo se ha reportado a FASECOLDA más de 1.000 eventos relacionados con enfermedad laboral siendo los sectores más representativos: Industria manufacturera, agricultura, inmobiliario, administración pública y defensa, servicios sociales y salud, financiero, comercio y educación.

En términos de pérdida de productividad agregada, a partir de la estrategia empírica anteriormente enunciada, se ha registrado una pérdida de 140.000 millones de pesos colombianos en el período 2009 - 2018 en Bogotá D.C. en términos de "Absenteeism" lo cual representa aproximadamente el $7 \%$ del Producto interno bruto (PIB) Total de Bogotá. Ahora bien, al ser este un proyecto de investigación en curso, para obtener resultados frente a "Presenteeism" será necesario ir más allá de las bases de datos actuales y hacerse un "Lab - in the field experiment", lo cual abre la posibilidad de nuevas investigaciones en este campo. 


\section{Conclusiones}

Se evidencia que el problema de investigación propuesto es relevante a nivel de la competitividad y productividad del país y el mundo. Por otro lado, los resultados aquí mostrados son exploratorios y por lo tanto es necesario realizar un tratamiento micro - econométrico más profundo y así ganar en precisión de los resultados obtenidos; dado que no se ha tomado en cuenta el "presenteeism" es probable que las pérdidas de productividad sean mayores.

A pesar de lo anterior, con los resultados obtenidos se considera muy oportuno y necesario diseñar políticas orientadas a revisar la salud física y mental de los trabajadores bogotanos, ya que trabajar más del tiempo óptimo (fisiológicamente) puede tener efectos negativos en el mediano y largo plazo. Finalmente, es necesario revisar las políticas de salud y seguridad en el trabajo, al ser actualmente más empleado - orientadas y no empleador- orientadas.

\section{Referencias}

[1] Chaker, L., Falla, A., van der Lee, S. J., Muka, T., Imo, D., Jaspers, L., ... Franco, O. H.. The global impact of non-communicable diseases on macro-economic productivity: a systematic review. European journal of epidemiology, (2015); 30(5), 357-395. doi:10.1007/s10654-015-0026-5

[2] Consejo Privado de Competitividad - CPC. Informe nacional de competitividad 2013 - 2014. [Consultado el 10 de mayo de 2020]. Disponible en: https://compite.com.co/informe/informe-nacional-decompetitividad-2013-2014/

[3] Jakubczyk, M. \& Koń, B. The impact of firms' adjustments on the indirect cost of illness, International Journal of Health Economics and Management, Springer, (2017), vol. 17(3), pages 377-394, September. doi: 10.1007/s10754-017-9212-1

[4] Kigozi, J., Jowett, S., Lewis, M. Estimating productivity costs using the friction cost approach in practice: a systematic review. European Journal of Health Economics, (2016), 17: 31. doi: https://doi.org/10.1007/s10198-014$0652-\mathrm{y}$

[5] Mitchell, R. J., \& Bates, P. Measuring health-related productivity loss. Population health management, (2011), 14(2), 93-98. doi:10.1089/pop.2010.0014

[6] Pearce, A.M., Hanly, P., Timmons, A., Walsh, P.M., O'Neill, C., O'Sullivan, E., Gooberman-Hill, R., Thomas, A.A., Gallagher, P., Sharp, L. Productivity losses associated with head and neck cáncer using the human capital and friction cost approaches. Applied Health Economics and Health Policy, Springer, (2015), vol. 13 (4), p. 359 - 367, August. doi: 10.1007/s40258-015-0155-8

[7] Petrie, J. G., Cheng, C., Malosh, R. E., VanWormer, J. J., Flannery, B., Zimmerman, R. K., ... Ohmit, S. E.. Illness Severity and Work Productivity Loss Among Working Adults With Medically Attended Acute Respiratory Illnesses: US Influenza Vaccine Effectiveness Network 2012-2013. Clinical infectious diseases: an official publication of the Infectious Diseases Society of America, (2015), 62(4), 448-455. doi:10.1093/cid/civ952

[8] Pearce, A; Et al. Productivity losses associated with head and neck cáncer using the human capital and friction cost approaches. Applied Health Economics and Health Policy, Springer, (2015b), vol. 13 (4), p. 359 - 367. doi: https://doi.org/10.1007/s10198-014-0652-y

[9] Suzuki, Tomoko; et al. Relationship between sickness presenteeism (WHO-HPQ) with depression and sickness absence due to mental disease in a cohort of Japanese workers. Journal of Affective Disorders, (2015), 180, p. 14 - 20. doi: 10.1016/j.jad.2015.03.034

[10] Wickramasinghe ND, Horton J, Darshika I, Galgamuwa KD, Ranasinghe WP, Agampodi TC, et al. Productivity cost due to postpartum ill health: A cross-sectional study in Sri Lanka. PLOS One 12, (2017), (10), p 1 - 12. doi: https://doi.org/10.1371/journal.pone.0185883

[11] Agampodi TC, et al. Productivity cost due to postpartum ill health: A cross-sectional study in Sri Lanka. PLOS One 12, (2017), (10), p 1 - 12. doi: https://doi.org/10.1371/journal.pone.0185883

[12] Zhang, W., Sun, H., Woodcock, S., \& Anis, A. H.. Valuing productivity loss due to absenteeism: firm-level evidence from a Canadian linked employer-employee survey. Health economics review, (2017), 7(1), 3. doi:10.1186/s13561-016-0138-y

[13] Zhang, Wei; Sun, Huiying; Woodcock, Simon; Aslam, Anis. Illness related wage and productivity losses: Valuing 'Presenteeism'. Social Science \& Medicine, (2015), $147, \quad$ p. 62 - $71 . \quad$ doi: https://doi.org/10.1016/j.socscimed.2015.10.056 